\title{
NUMERICAL STUDY OF THE EFFECT OF SURFACE WETTABILITY ON PERFORMANCE OF THE SPRAY COOLING PROCESS
}

\author{
R. ATTARZADEH \& A. DOLATABADI \\ Department of Mechanical and Industrial Engineering, Concordia University, Montreal, Canada.
}

\begin{abstract}
The process of cooling caused by a water droplet contacting a surface has been extensively reported in the literature; however, the effect of surface wettability on the outcome of the cooling rate has yet to be analyzed. Due to optical limitations inside a liquid droplet, a three-dimensional (3D) computational fluid dynamics (CFD) model, including coupling between multiphase flow and the conjugated heat transfer module was developed to simulate the impact, spreading and transient heat transfer between a cold-water droplet and a heated surface. The total heat transfer results were calculated for both superhydrophobic and hydrophilic surfaces. The Navier-Stokes equation expressing the flow distribution of the liquid and the gas, coupled with the volume of fluid (VOF) method for tracking the liquid interface, was solved numerically using the finite volume methodology. The grid dependency test was examined for the 3D model, even though the convergence of the results was not exact. The $2 \mathrm{~mm}$ diameter water droplet with the Weber numbers 7, 25 and 62, which correspond to non-splashing regimes, were impinged onto two different surfaces. We showed that spray cooling on a superhydrophobic substrate was capable of improving the efficiency of the cooling process up to $40 \%$ compared to that of a hydrophilic surface. Additionally, the critical Weber regime was obtained for the optimal heat transfer between the droplet and the two substrates.

Keywords: Cooling process, Superhydrophobic, Hydrophilic, Droplet impact
\end{abstract}

\section{INTRODUCTION}

The impingement of spraying cold drops onto heated dry walls, and the associated heat transfer mechanism occurs in many engineering applications such as, spray drying [1], internal combustion engines [2], spray coating [3], and spray cooling processes [4]. Spray cooling is a technique of increasing interest for cooling of electronic and mechanical elements, and is characterized by a high rate of heat transfer, uniformity of heat removal, and low droplet impact velocity. In spray cooling process, spray of small droplets on a heated surface is performed to remove large amounts of energy by taking the advantage of substantial convective heat transport through impinging droplets [1]. The ability of the liquid coolant to change phase in constant temperature, and at the same time, to dissipate heat in the form of latent heat makes the cooling process very interesting.

The fluid dynamics of impinging drops and the liquid film formation is complicated, and requires fundamental study. In addition, the mechanism by which heat is removed during spraying are poorly understood, and because of its dependence on many parameters that are not easily varied dependently, predictive capabilities are quite limited.

In order to overcome aforementioned complications (droplet coalescence, liquid thin film formation, or phase change due to evaporation), a fundamental study of the spraying process, which is the impact of a single droplet onto a heated surface under non-fragmentation Weber threshold is carried out. Experimentally, it is shown that hydrophobic surfaces would have higher potential for removing thermal energy when are subjected to cooling spray, but not many detailed study was carried for better understanding the mechanics associated with this phenomena. 


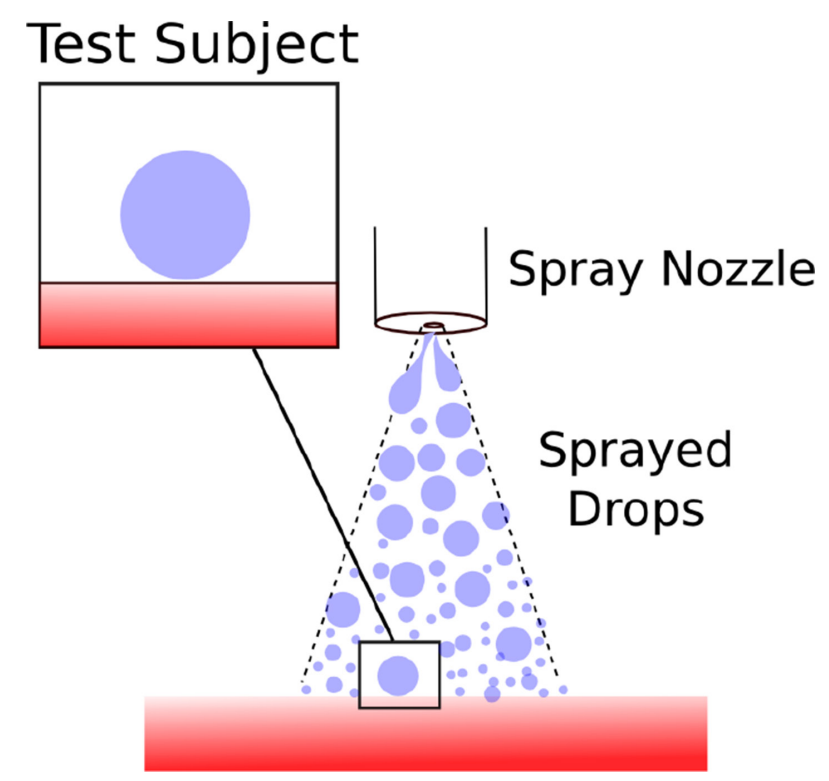

Figure 1: Schematic of sprayed drops in spray cooling process.

The primary objective of this study is to simulate the impact of a cold-water droplet on two preheated Aluminum substrates with infinitely thin hydrophilic and superhydrophobic coating through conjugated temperature gradient platform. We simulated $2.0 \mathrm{~mm}$ cold-water droplet as it impinges on the heated substrate, and measured the amount of thermal energy absorbed by the droplet after impact. The initial substrate temperature was set at $360^{\circ} \mathrm{K}$; slightly below the boiling temperature to avoid complication associated with the evaporation or phase change. Impact velocities ranged from 0.5 to $1.5 \mathrm{~m} . \mathrm{s}^{-1}$ to avoid fragmentation as the result of impact. Fluid flow and heat transfer during droplet impact were modeled using the Volume of Fluid (VOF) method.

\section{METHODOLOGY}

In this paper, a three dimensional (3D), multi-region conjugate heat transfer module is developed to analyze the mechanism in which heat is transferred between solid and fluid. Separate governing equations for each region are solved depending on their phase and the boundary interface.

\subsection{Governing equations}

In the fluid region, the Navier-Stokes equation expressing the flow distribution of the liquid and the gas, coupled with the Volume of Fluid (VOF) method for tracking the interface between the liquid and the gas is solved numerically using finite volume methodology. In VOF method [5], tracking of the interface is modeled by solving continuity equation for one of the two phases in each computational cell at every time step. The governing equations for the mass, momentum and energy balance in each fluid phase and on the interface, can be expressed as, 


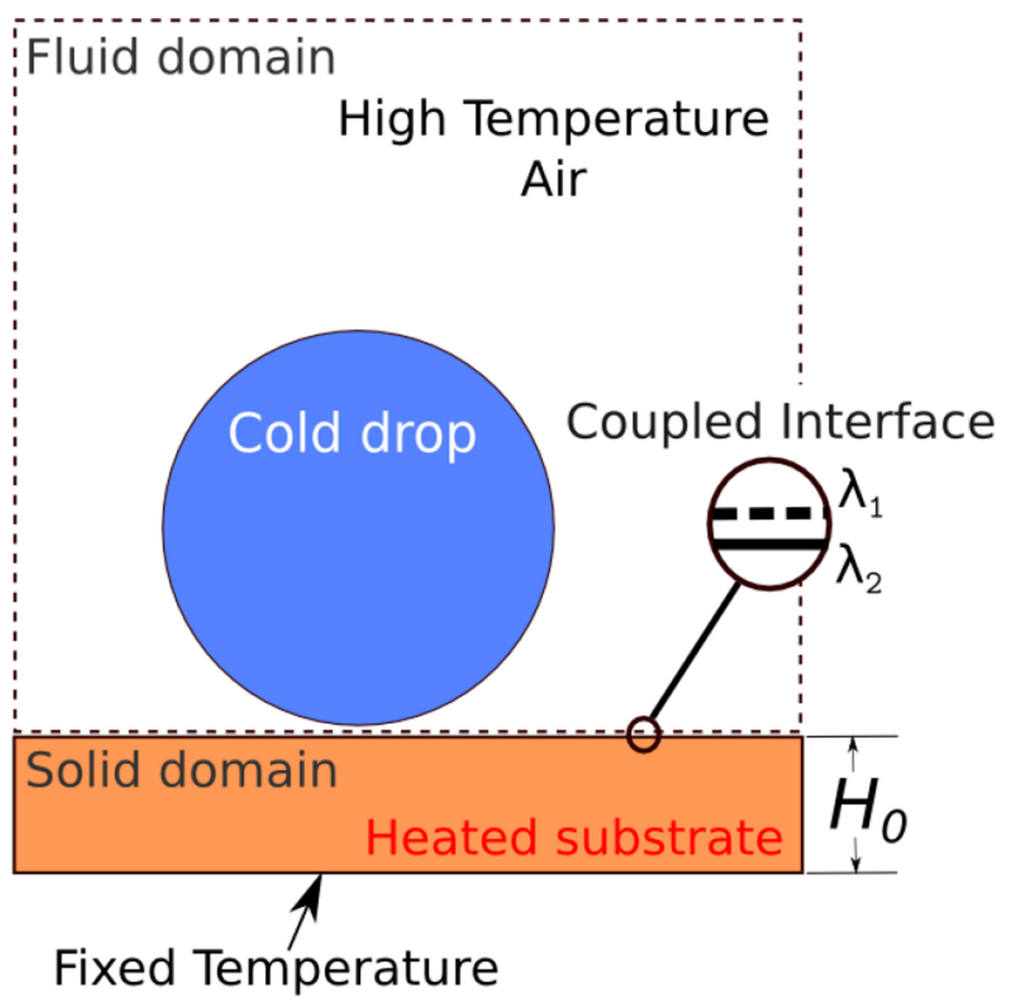

Figure 2: $3 \mathrm{D}$ computational domain, cold droplet and the $H_{0}=3 \mathrm{~mm}$-thick heated Aluminum substrate. $\lambda_{a} \& \lambda_{2}$ are the coupling interfaces.

$$
\begin{gathered}
\vec{\nabla} \cdot \vec{V}=0 \\
\frac{\partial \rho \vec{V}}{\partial t}+\vec{\nabla} \cdot(\rho \vec{V} \vec{V})=-\vec{\nabla} p+\nabla \cdot(\mu \nabla \vec{V})+\vec{F}_{b} \\
\frac{\partial \rho C p T}{\partial t}+\vec{V} \vec{\nabla} \cdot\left(\rho C_{p} T\right)=\nabla \cdot(k \nabla T)
\end{gathered}
$$

where $\boldsymbol{T}$ is temperature, $\boldsymbol{t}$ is time, $\vec{V}$ is the velocity vector, $\boldsymbol{p}$ is the pressure, $\vec{F}_{b}$ is the body force, and $a$ is the volume fraction which is used to calculate the viscosity $(\mu)$, density $(\rho)$, heat capacity $\left(C_{p}\right)$ and thermal conductivity $(K)$ as weighted averages based on the distribution of $\alpha$,

$$
\begin{gathered}
\rho=\alpha \rho_{I}+(1-\alpha) \rho_{g} ; \mu=\alpha \mu_{l}+(1-\alpha) \mu_{g} \\
K=\alpha K_{I}+(1-\alpha) K_{g} ; \rho C_{p}=\alpha \rho_{I} C_{p I}+(1-\alpha) \rho_{g} C_{p g}
\end{gathered}
$$

where the subscripts $\boldsymbol{l}$ and $\boldsymbol{g}$ denote the liquid and gas phases, respectively. In VOF method, the phase fraction scalers tracer $\alpha$, takes values between 0 and 1 . When a cell is empty with no traced fluid inside, the value of $a$ is zero; when the cell is full, $a$ is equal to 1 ; and when 
there is a fluid interface in the cell, $0<\alpha<1$. As the interface deforms with time, The evolution of fluid is governed by the transport equation,

$$
\frac{\partial \alpha}{\partial t}+(\vec{V} \vec{\nabla}) \alpha+\vec{\nabla} \cdot\left(\vec{V} \cdot\left(\vec{V}_{r} \alpha(1-\alpha)\right)=0\right.
$$

where $\vec{V}_{r}$ is the vector of relative velocity, and the last term is called artificial compression term contains the compression velocity, which is computed to avoid smearing of the phase interface [6].

$$
\vec{V}_{r}=n \min \left[C_{a} \frac{|\phi|}{\left|S_{f}\right|}, \max \left(\frac{|\phi|}{\left|S_{f}\right|}\right)\right]
$$

where $C_{a}, \boldsymbol{n}, \varphi$, and $S_{f}$, are the compression coefficient, unit normal flux, face volume flux and cell face area vector respectively. The interface unit normal $\vec{n}$ is computed by taking the gradient of smoothed volume fraction $\alpha$ at the cell faces,

$$
\vec{n}_{f}=\frac{\vec{\nabla} \alpha}{|\vec{\nabla} \alpha|+\delta}
$$

where $\delta$ is a small number to stabilize the calculation in regions outside the transition region where $\mid \vec{\nabla} \alpha \rightarrow 0$. In this study, $\delta=10^{-8}$ is used for the entire simulations. The main benefit of such formulation is in the possibility of capturing the sharp interface region. The continuum surface force method [7] is used to model surface tension as a body force $\vec{F}_{b}$ that acts only on interfacial cells,

$$
\vec{F}_{b}=\sigma k \vec{\nabla} a \quad \& \quad k=-\vec{\nabla} \cdot\left(\frac{\vec{\nabla} a}{|\vec{\nabla} a|}\right)
$$

where $\sigma$ is the surface tension, and $k$ is the mean curvature of the free surface.

The pressure-implicit with splitting operators (PISO) scheme in transient flow is used for the pressure-velocity coupling. The wettability effects on the substrate are taken into account by implementation of Kistler's dynamic contact angle model [8].

$$
\theta_{d}=f_{H}\left[C a+f_{H}^{-1}(\theta e)\right],
$$

where $\mathrm{Ca}$ is the capillary number, $f_{H}$ the Hoffman's function,

In the above equation, the equilibrium contact angle $\theta_{\mathrm{e}}$ is replaced by either the advancing contact angle, $\theta_{A}$, or the receding contact angle, $\theta_{R}$, depending on the direction of the velocity vector at the contact line or the static contact angle, $\theta_{\mathrm{S}}$, if the contact line velocity is zero. Among no-slip models, Kistler's correlation [8] have been extensively used to investigate droplet dynamics, and proven to be in good agreement with the experiment observation [9]. In addition, it is shown that this model is capable to capture the relevant physics of dropletsubstrate interaction and provides good agreement with experiment.

The heat transfer in solid substrate can be modeled by solving the Laplace equation for the pure conductive heat transfer in a solid substrate:

$$
\rho_{s} C_{s} \frac{\partial T_{s}}{\partial t}=\vec{\nabla} \cdot\left(k_{w} \vec{\nabla} T_{s}\right)
$$




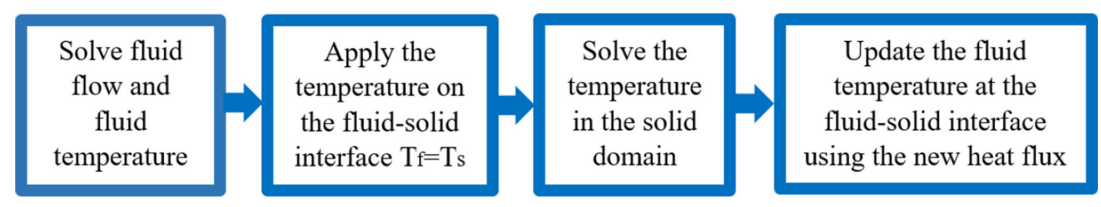

Figure 3: Iterative solution procedure of domains coupling.

Table 1: Material properties.

\begin{tabular}{lllll}
\hline & $\rho\left(\frac{k g}{m^{3}}\right)$ & $K\left(\frac{k g . m}{s^{3} \cdot T}\right)$ & $C_{p}\left(\frac{m^{2}}{s^{2} \cdot T}\right)$ & $v\left(\frac{m^{2}}{s}\right)$ \\
\hline Air & 1 & 0.02 & 1,000 & $1.78 \times 10^{-5}$ \\
Water & 1,000 & 0.6 & 4,200 & $1.0 \times 10^{-6}$ \\
Alumin & 2,700 & 205 & 900 & - \\
\hline
\end{tabular}

The lower surface of the substrate is subjected to fixed temperature boundary condition $\left(360^{\circ} \mathrm{K}\right)$. The substrate density, thermal conductivity and heat capacity are given in Table 1 .

\subsection{Regions coupling}

There are two approaches for solving multi-region problems; monolithic and partitioned. The monolithic approach seeks to define a series of partial differential equations (PDEs) that govern the entire fluid and solid domain and then discretize the complete domain. While theoretically simple, this approach is difficult to actually implement due to the differing mathematical and numerical properties of the two domains. On the other side, the partitioned approach seeks to utilize existing fluid and solid solutions and couple these solvers at the interface of the two domains. The fluid solver uses a separate set of equations, variables and mesh than the solid solver. The coupling happens at the boundary $\left(\lambda_{a} \& \lambda_{2}\right)$ where the temperature of the fluid updates the boundary of the solid which is then solved for to determine the gradient temperature at the interface boundary. This updated boundary is then used to solve the fluid domain. As this method solves different sets of matrices, parallelism of the computation is more efficient. In this paper, the portioned coupling method was used to couple solid and fluid domains. Considering the governing equations for each domain, the temperature and heat fluxes should be equal at the interface,

$$
T_{f}=T_{s} \quad \& \quad K_{f} \nabla T_{f}=K_{s} \nabla T_{s}
$$

where, $T_{f}$ and $T_{s}$ are the temperatures of fluid and solid domains, respectively. Boundary conditions are applied on boundary patches. Each domain solving module is placed inside a separate loop to meet the coupled convergence through a global iterative process.

Validation of the solver is performed using one-dimensional, steady state situation where heat is transferred between gas and wall to ensure the calculation of the coupled interface is correct [10]. It is basically a wall, with hot and warm air flowing next to it, which results in a temperature gradient in the wall. In a 1D problem, heat transfer occurs only in one direction. The results obtained from the model and the analytical study were in close agreement with less than $1 \%$ divergent. 


\section{GEOMETRICAL CONFIGURATION AND MESHING}

The computational domain size of $16 \mathrm{~mm} \times 20 \mathrm{~mm} \times 14 \mathrm{~mm}$ is used for the 3D simulation of the impinging droplet onto heated substrate. The domain is discretized by about 1 million, 2 million, and 3.5 million Hexahedral mesh with adaptive refinement at the interfacial cells. The mesh dependency test is performed based on the jumping velocity of a droplet on superhydrophobic surface. As the change of droplet jumping velocity in 3.5 million and 2 million mesh was minimal, the domain composed of 2 million mesh is applied in this study. The cells are refined so to have 60 cells per diameter of the droplet before impingement. The substrate thickness of $3 \mathrm{~mm}$ is extracted from the domain. The exterior surface of solid region that is in common with fluid is coated with hydrophilic $\left(15^{\circ}\right)$ and superhydrophobic $\left(180^{\circ}\right)$ materials. The material properties of the substrate are given in Table 1.

\section{RESULTS AND DISCUSSION}

In order to study, the effect of surface wettability on the transient heat transfer and total heat transfer between the droplet and the surface, the impingement of water droplets onto two surfaces with different wettabilities was carried out.

Figure 4 shows the time lapse of the droplet dynamics during the impingement on infinitely thin superhydrophobic and hydrophilic coatings on aluminum substrates. We found that the hydrodynamics of the liquid drop changed depending on the wettability of a surface. Because of the extremely low-surface energy of the superhydrophobic coating, the droplet detached a certain amount of time after impingement, called the droplet contact time [11]. According to Richard [11], the contact time is not a function of impact velocity. Instead, it is a strong function of the surface energy and directly proportional to the liquid density, the droplet size and the liquid surface energy. For the sake of simplicity in analysis, the physical times of all simulations were bounded according to the droplet contact time on the superhydrophobic surface. Previous research showed that the contact time does not depend on the Weber number [11]. For droplets as large as $2 \mathrm{~mm}$ in diameter, the contact time scaled with the inertia-capillary timescale $\left(T=\sqrt{\frac{\rho^{*} R^{3}}{\sigma}}\right)$, and for high deformation impact (non-splashing regime) on a superhydrophobic surface, the contact time was approximated as $t_{\mathrm{c}}=2.3^{*} T$.

As shown in Fig. 4a, the droplet spread on the hydrophilic substrate and covered a higher surface area compared to the same size droplet impinging on the superhydrophobic surface. According to the law of conductive heat transfer, higher surface area contact should result in a higher heat transfer rate between the two media.

On the superhydrophobic surface, the droplet spread up to its maximum spreading diameter. At the same time, the kinetic energy of the impact transformed into surface energy in the form of potential energy. At a point in time after this occurred, the droplet released its excess potential energy and started to recoil. Once the recoiling stage was over, the droplet completely rebounded and detached from the substrate. The temperature of the droplet and surface changed according to the heat transfer rate between the two media during the contact event.

In order to measure and compare the total heat transfer between the hydrophobic and hydrophilic aluminum surfaces, the total energy of the droplet before and after impact were 


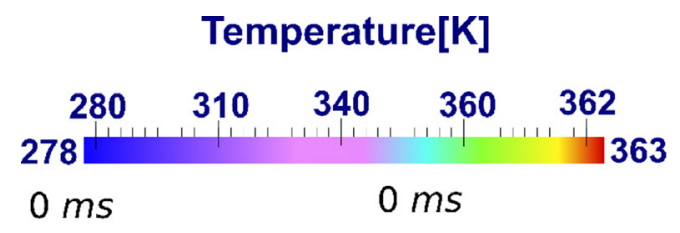

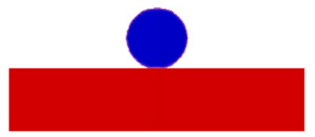

$1 \mathrm{~ms}$

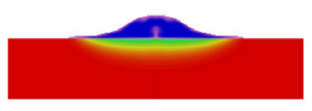

$2 \mathrm{~ms}$

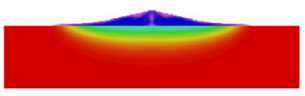

$3 \mathrm{~ms}$

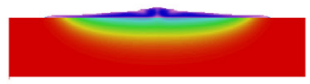

$5 \mathrm{~ms}$

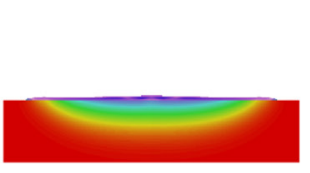

$7 \mathrm{~ms}$

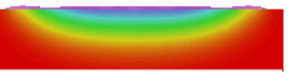

$9 \mathrm{~ms}$

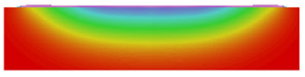

(a)

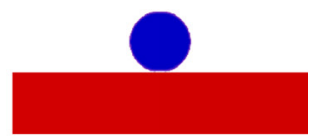

$1 \mathrm{~ms}$

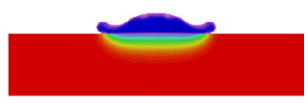

$2 \mathrm{~ms}$

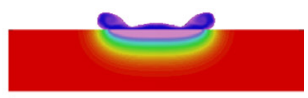

$3 \mathrm{~ms}$

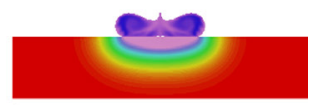

$5 \mathrm{~ms}$

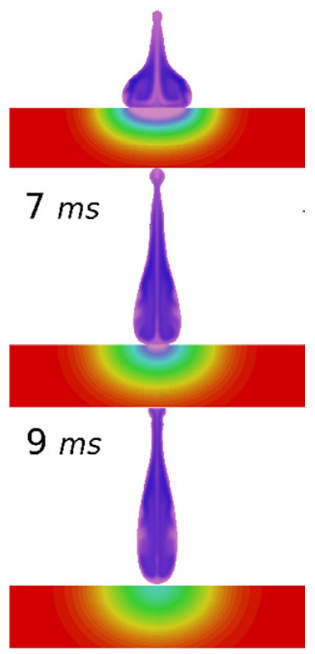

(b)

Figure 4: Impact of $2 \mathrm{~mm}$ water droplet on (a) hydrophilic and (b) superhydrophobic heated aluminum substrate, $V_{\mathrm{d}}=1 \mathrm{~m} \cdot \mathrm{s}^{-1}$. 


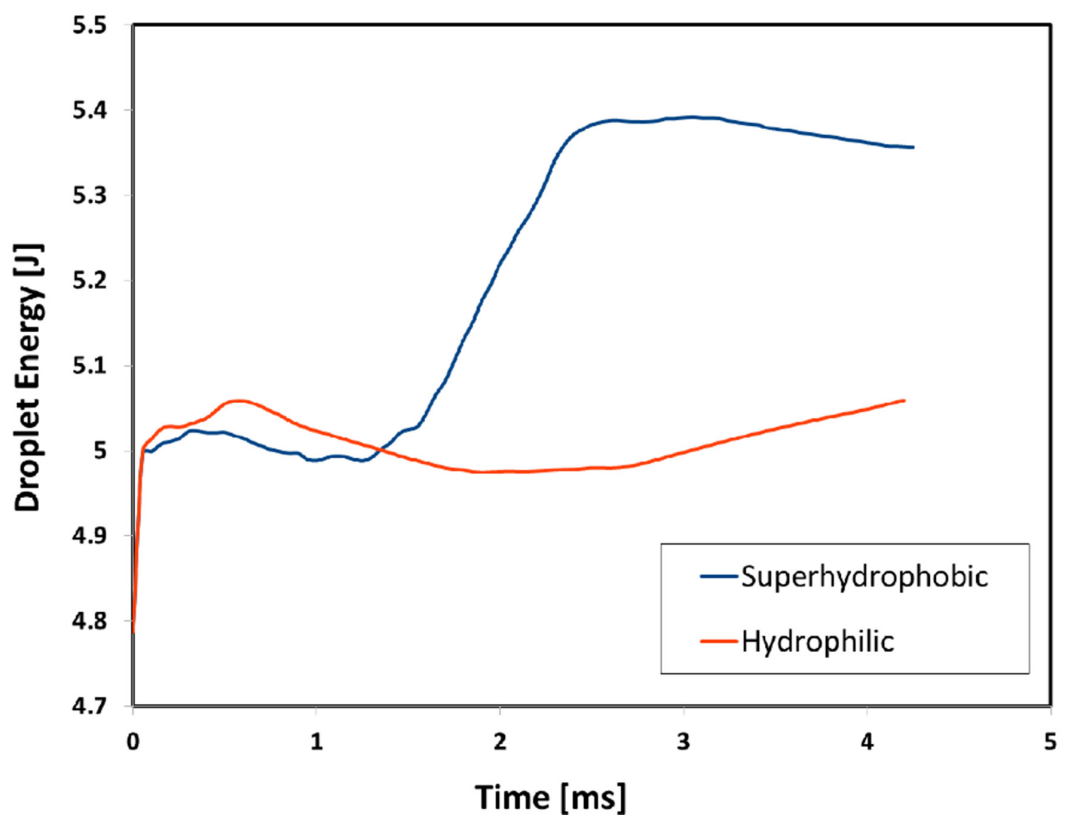

Figure 5: Energy in transit between droplet and substrates, $V_{d}=1 \mathrm{~m} \cdot \mathrm{s}^{-1}$.

calculated for both surfaces (Fig. 5). Interestingly, the total energy transferred to the superhydrophobic surface was higher than that transferred to the hydrophilic surface, despite the smaller contact area during the impingement event.

We observed strong mixing on the periphery of the droplet lamella on the superhydrophobic surface during its maximum spreading stage (Fig. 6), which resulted in a higher heat transfer rate between the droplet and the heated aluminum substrate. The lower volume of droplet lamella that was initially in contact with the heated aluminum was heated by the surface, and, due to a high intensity of mixing, the upper and lower volumes circulated until a thermal equilibrium condition was nearly met when the droplet detached from the substrate.

The dynamics of the droplet on the hydrophilic surface was different. The mixing associated with the recoiling stage was not observed in the droplet lamella. The droplet spread and the upper volume of the droplet temperature was raised gradually due to convective heat transfer with air. Figure 7 shows the total energy enhanced by the droplet at the time of detachment for various Weber numbers by changing the terminal velocity of the impact. Although the Weber number was reported not to change the contact time of the droplet on the superhydrophobic substrate, We found that it can have a significant effect on the mixing intensity, and consequently the amount of heat transferred between the solid substrate and the liquid droplet. As the dimensionless Weber number increased, the droplet obtained more thermal energy from the surface. The total amount of absorbed energy was greater on the superhydrophobic surface than on the hydrophilic surface. The best cooling performance in the non-fragmentation regime was observed on the superhydrophobic surface for the Weber number near the fragmentation threshold. 


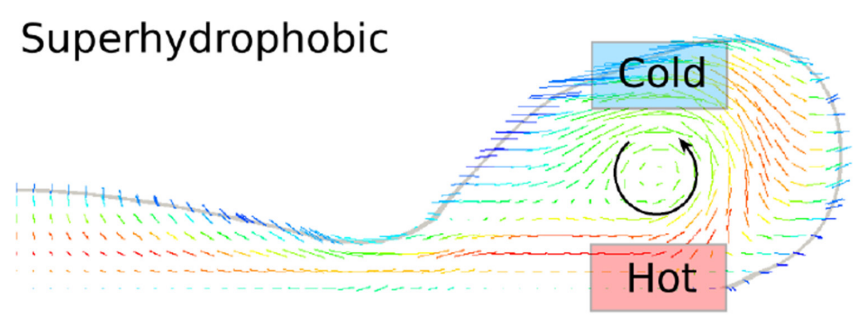

\section{Hydrophilic}

Figure 6: High intensity mixing of liquid lamella and higher heat transfer rate of superhydrophobic comparing hydrophilic surface, $V_{d}=1 \mathrm{~m} \cdot \mathrm{s}^{-1}$.

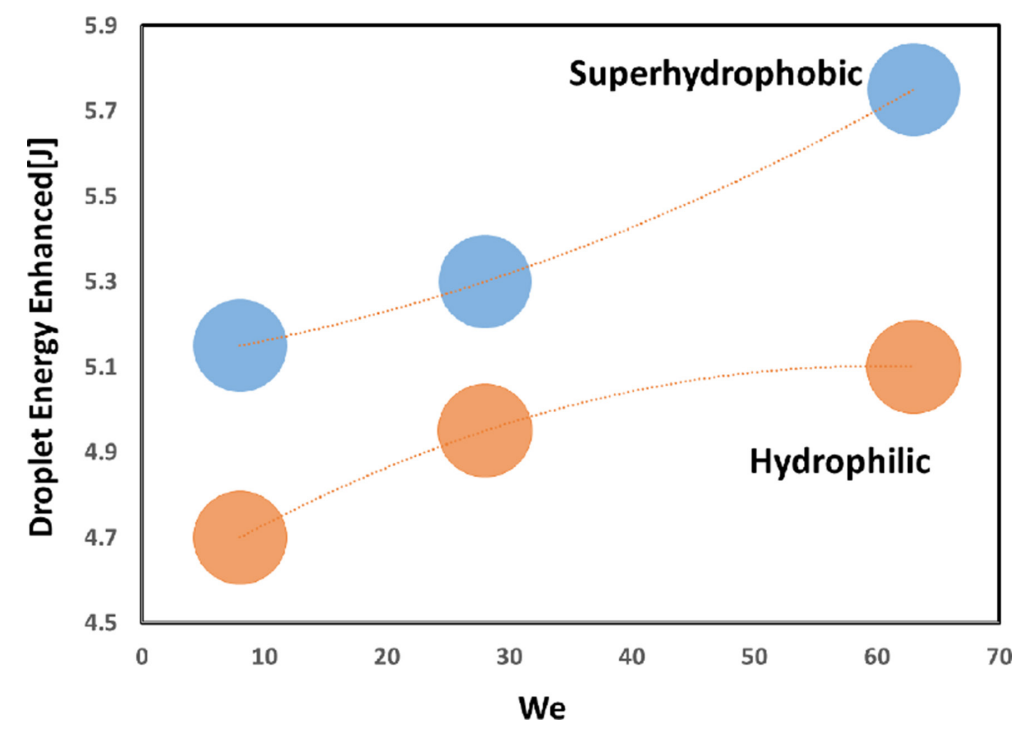

Figure 7: Thermal energy of $2 \mathrm{~mm}$ droplet at the time of detachment for three Weber numbers.

\section{CONCLUSION}

The conjugate temperature gradient multi-phase model was developed to simulate the impact of a cold-water droplet on a heated aluminum substrate with infinitely thin coatings. We showed that the spray cooling effect on the superhydrophobic substrate improved the efficiency of the process up to $40 \%$ compared with spray cooling on a hydrophilic surface. This efficiency increase was associated with a high mixing intensity of the liquid inside the droplet lamella, which increased the heat transfer rate with the substrate. Additionally, we found that the Weber number has a significant impact on the cooling rate of the surface.

\section{ACKNOWLEDGEMENT}

The authors gratefully acknowledge the financial support from Natural Sciences and Engineering Research Council of Canada (NSERC). 


\section{REFERENCES}

[1] Xie, J.L., Gan, Z.W., Duan, F., Wong, T.N., Yu, S.C.M. \& Zhao, R., Characterization of spray atomization of pressure swirl nozzles. International Journal of Thermal Sciences, 68, pp. 94-102, 2013.

http://dx.doi.org/10.1016/j.ijthermalsci.2012.12.015

[2] Kim, J., Spray cooling heat transfer: The state of the art. International Journal of Heat and Fluid Flow, 28(4), pp. 753-767, 2007.

http://dx.doi.org/10.1016/j.ijheatfluidflow.2006.09.003

[3] Girard, F., Meillot, E., Vincent, S., Caltagirone, J.P. \& Bianchi, L., Contributions to heat and mass transfer between a plasma jet and droplets in suspension plasma spraying. Surface and Coatings Technology, 268, pp. 278-283, 2015. http://dx.doi.org/10.1016/j.surfcoat.2015.01.011

[4] Su, T., Chang, C., Reitz, R.D., Farrell, P., Pierpont, A. \& Tow, T., Effects of injection pressure and nozzle geometry on spray SMD and D.I. emissions. Technical report, SAE Technical Paper, 1995.

[5] Hirt, C.W. \& Nichols, B.D., Volume of fluid (VOF) method for the dynamics of free boundaries. Journal of Computational Physics, 39(1), pp. 201-225, 1981. http://dx.doi.org/10.1016/0021-9991(81)90145-5

[6] Rusche, H., Computational fluid dynamics of dispersed two-phase flows. Ph.D. thesis, Imperial College London (University of London), 2003.

[7] Bussmann, M. \& Mostaghimi, On a three-dimensional volume tracking model of droplet impact. Physics of Fluids, 11(6), p. 1406, 1999.

[8] Kistler, S.F., Hydrodynamics of wetting. Wettability, 49, p. 311, 1993.

[9] Farhangi, M.M., Graham, P.J., Choudhury, N.R. \& Dolatabadi, A., Induced detachment of coalescing droplets on superhydrophobic surfaces. Langmuir, 28(2), pp. 1290-1303, 2012. http://dx.doi.org/10.1021/la203926q

[10] Bergman, T.L., Incropera, F.P., DeWitt, D.P. \& Lavine, A.S., Fundamentals of Heat and Mass Transfer, John Wiley \& Sons, 2011.

[11] Richard, D., Clanet, C. \& Queré, D., Surface phenomena: Contact time of a bouncing drop. Nature, 417(6891), pp. 811-811, 2002.

http://dx.doi.org/10.1038/417811a 